\title{
Post-Acute Sequelae of COVID-19 and Polypharmacy: If You Think There's Too Much Polypharmacy Now, Just Wait
}

\author{
Demetra Antimisiaris, PharmD, BCGP, FASCP ${ }^{1 *}$ \\ ${ }^{1}$ Center of Excellence for Research in Infectious Diseases, University of Louisville, Louisville, KY, USA \\ *demetra.antimisiaris@louisville.edu
}

Recommended Citation: Antimisiaris D. Post-acute sequelae of COVID-19 and polypharmacy: if you think there's too much polypharmacy now, just wait. Univ Louisville J Respir Infect 2021; 5(1):Article 19.

\section{The perfect storm}

The editor-in-chief of the Lancet, Richard Horton, recently called the intersection of the COVID-19 pandemic with the epidemic of increasing chronic disease burden an "acute-on-chronic health emergency".[1] The Institute for Health Metrics and Evaluation at the University of Washington describes the rising worldwide chronic disease burden and public health failures as "fueling" the COVID-19 pandemic.[1, 2] Prior to the pandemic, polypharmacy per capita was steadily increasing over decades due to many factors, but especially because of increased chronic disease burden among the general population worldwide. $[3,4]$

\section{Chronic disease, and more post-COVID-19 chronic disease}

We are learning that post-acute sequelae of COVID19 (PASC) in many ways parallel post-intensive care syndrome (PICS), with persistent physical, cognitive and psychological sequelae impacting quality of life.[5] This implies that many recovered COVID-19 patients will live with increased disease burden and accompanying polypharmacy, in addition to their pre-COVID19 conditions and medications. The wide variety of PASC reported within the first year of the pandemic indicates that PASC patients will experience an abrupt increase in per patient burden of diagnoses in the near term and possibly permanently.[6]

In addition to PASC patients, there are an unknown number of milder COVID-19 infection cases. Regardless of the severity of infection, it is clear that prolonged after-effects of COVID-19 after treatment or hospitalization do exist.[7] Johansson et al. report that even outpatient COVID-19 infection cases can have non-specific symptoms of autonomic dysfunction, blood pressure dysregulation, and orthostatic intolerance (postural orthostatic tachycardia syndrome, or POTS).[8] Multiple neurological sequelae of COVID-19 infection have also been reported, including cerebrovascular disorders (stroke, vasculitis), altered mental status (encephalitis, encephalopathy, seizures), peripheral nervous system problems (myositis, Guillain-Barre syndrome), movement disorders (ataxia, myoclonus and opsoclonus), and neuropsychiatric problems (depression, personality change, mania, psychosis, and catatonia).[9]

In March 2021, results were published from an ongoing prospective study in the United Kingdom with a cohort of 201 community-based low-risk post-COVID19 patients (mean age 45; range 21-71). The patients reported fatigue $(98 \%)$, muscle aches $(87 \%)$, breathlessness $(88 \%)$, and headaches $(83 \%)$. Mild organ impairment was present in the heart $(26 \%)$, lungs $(11 \%)$, kidneys $(4 \%)$, liver $(28 \%)$, pancreas $(40 \%)$, and spleen $(4 \%)$, with single organ $(70 \%)$ and multiorgan (29\%) impairment. In this study, severe PASC was associated with radiological evidence of cardiac damage.[10]

\section{Yet another perfect storm}

The intersection of the COVID-19 pandemic with the epidemic of increasing chronic disease burden worldwide has been called "the perfect storm".[1] The expected burden of additional diagnosis in post-COVID19 patients, with high antecedent chronic disease burdens and accompanying polypharmacy, is yet another perfect storm, especially for the most vulnerable COVID-19 patients: older persons.[11] Older adults tend to have multiple comorbidities and have the highest level of polypharmacy use in society, even preCOVID-19.[12] The prevalence of polypharmacy increases proportionally with increasing age.[12] Older 
persons are seven times more likely to be hospitalized for adverse drug events than younger persons.[13] In the United States and Europe, the number of people taking five or more prescription drugs (not counting over-the-counter medications, herbal supplements, and vitamins) is increasing rapidly. One study reported that $85 \%$ of older adults with cancer received five or more prescription medications, and $43 \%$ received ten or more.[14] Cancer.org reports the risk of living with cancer in adults 85 years and older is 1 in 6 for men and 1 in 8 for women, and this age cohort is expected to triple by 2060.[15] Older adults typically live with chronic subclinical systemic inflammation, a heightened baseline inflammatory status termed "inflammaging." Inflammaging is thought to start in middle age and clinically presents as excessive chronic disease. Inflammaging is associated (causally in many cases) with age-related diseases, such as insulin resistance, cardiovascular disease, osteoarthritis, COPD, emphysema, pulmonary arterial hypertension, Alzheimer's disease, Parkinson's disease, macular degeneration, sarcopenia, immunosenescence, and frailty.[16]

Hyperinflammatory syndromes-such as SARS-CoV2 (COVID-19), ARDS, cytokine storm syndrome, and PICS-are associated with short- and long-term sequelae following recovery. These syndromes lead to an accelerated state of chronic subclinical inflammation and an increase or worsening of age-related conditions, including frailty in older (as well as potentially younger) persons with PASC who are already living with high chronic disease burden.[16] Medical scholars expect post-COVID-19 patients to experience accelerated inflammaging.

It is not difficult to see how polypharmacy levels will dramatically increase due to the COVID-19 pandemic, given the anticipated burden of PASC conditions and the potential for accelerated inflammaging. Clinicians are already anxious about the impact of added neurocognitive and psychiatric conditions that result from heavy burdens of medical comorbidities. Layering neuropsychiatric problems on top of chronic metabolic and inflammatory disease presents a difficult and complicated medication management challenge.[17] Additionally, medications can interfere with clinical tests and outcomes.[18, 19]

Consider the case scenario of polypharmacy in an older male patient with multiple chronic diseases, discharged from hospital after acute COVID-19 infection, who now has seizures and survived an acute myocardial infarction due to the COVID-19 infection. The patient now lives with anxiety, insomnia, and depression because of post-acute COVID-19 change in functional status and an increased level of frailty (Figure 1).[20, 21]

This patient was discharged with a new prescriptions for a beta blocker (metoprolol), because he had an acute myocardial infarction, and levetiracetam for new-onset seizures also caused by the acute COVID-19 infection. The patient was also discharged with a tramadol prescription for persistent pain. Two months after hospital discharge, because the patient became depressed about diminished post-acute COVID-19 functional status, he was prescribed fluoxetine by his community physician. The levetiracetam was dosed too high for the patient's renal function, resulting in restlessness and psychiatric adverse effects. The patient's family took the patient to the grocery store easy-access clinic for symptoms of insomnia, where he was prescribed trazodone. An acute COVID-19 episode can cause post-COVID-19 sequelae that significantly increase a patient's polypharmacy burden. Increased polypharmacy burden increases the risk of adverse events, and for this patient, there are several new medication burden-related threats. The tramadol may not be effective because fluoxetine inhibits conversion to active drug; fluoxetine also inhibits metabolism of active metoprolol, which may put the patient at risk of bradycardia and hypotension. The fluoxetine may interact with the patient's existing simvastatin prescription and cause muscle pain or rhabdomyolysis. Furthermore, levetiracetam toxicity may be unrecognized and misdiagnosed as new-onset psychosis or dementia and start a prescribing cascade to address medication-induced symptoms.

\section{Significance of expected post COVID-19 syndrome hyper-polypharmacy}

The potential for hyper-polypharmacy worldwide due to the COVID-19 pandemic is significant. At a time when societies are trying to gain control over rampant polypharmacy with large government-sponsored programs, such as "Polypharmacy Management by 2030" in the European Union, the "Canadian Deprescribing Network," the "US Deprescribing Research Network," and Australia's "National Prescribing Service Medicinewise," it is daunting to think about the potential for added medication load per capita around the world due to PASC and post-outpatient COVID-19 syndrome.[12, 22-24] Well-intentioned medication use in 2016 was responsible for 275,689 deaths and cost $\$ 528.4$ billion dollars.[25] This means that if medication-related problems were a disease counted by the Centers for Disease Control as a cause of death in the United States, it would rank as the third leading cause of death behind cancer and heart disease, but ahead of diabetes, Alzheimer's disease and others.[26] Most medication-related problems are avoidable with the optimization of adherence, prescribing practices, and, most importantly, monitoring.[27, 28] The more complex the patient and the higher the number of medications, supplements and vitamins used concomitantly, the more challenging medication use optimization becomes. Therefore, the COVID-19 pandemic may 


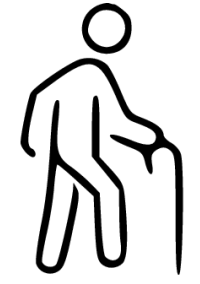

\begin{tabular}{|l|}
\hline Pre-PASC Diagnoses \\
Diabetes \\
Hypertension \\
Severe osteoarthritis \\
Chronic renal failure \\
Pre-Pasc Medications \\
Glipizide \\
Losartan \\
Aspirin (81mg) \\
Tramadol \\
Simvastatin \\
\hline
\end{tabular}

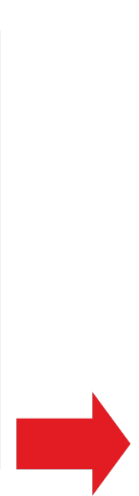

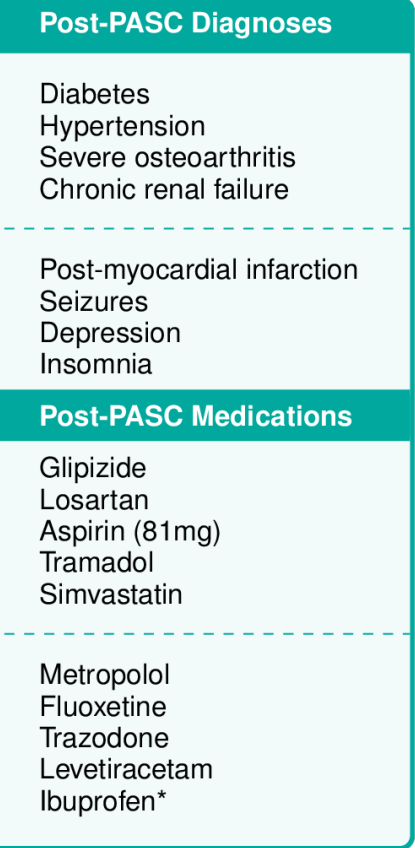

Post-PASC Drug-Drug Interactions

Tramadol + Fluoxetine: Tramadol is a prodrug; fluoxetine inhibits conversion to active drug.

Metoprolol + Fluoxetine: Fluoxetine inhibits metabolism of metorprolol $\Rightarrow$ bradycardia

\section{Post-PASC Drug-Disease Interactions}

Levetiracetam requires strict renal dose adjustment: risk irritable behavior and psychiatric adverse effects (+ addition of further medications).

\section{Post-PASC Drug-Induced Problems}

Simvastatin metabolized by CYP 3A4 pathway + fluoxetine: increased simvastatin levels and muscle pain. (Selfprescribed ibuprofen causes further renal impairment and further medication toxicity)

Figure 1. Scenario of an older adult with multiple chronic conditions, pre- and post-PASC. In addition to several new comorbidities, including added neurologic and psychiatric diagnoses, his added polypharmacy introduces an entire cascade of new problems.

*Self-prescribed. 
in time raise the death toll and costs of non-optimized medication use well beyond the third leading cause of death and $\$ 528$ billion. Additionally, the added layers of complex psychoactive and chronic disease management medications will make adverse drug-drug and drug-disease interactions, as well as toxidromes, more common, posing a difficult clinical management scenario for clinicians.

\section{Conclusion}

PASC patients are beginning to be characterized by biomedical scholars. It would be of value to char- acterize, quantify, and study approaches to the expected increase in rates of polypharmacy and potential cascade of new medication-related problems. From a public health and biomedical science perspective, polypharmacy has been as difficult a problem to tackle as the growing burden of chronic disease. Unmanaged polypharmacy is very costly in terms of morbidity and mortality, as well as monetarily. Tracking the count of pre-COVID-19 infection and post-COVID-19episode medication burden, as well as characterization of medications used to treat post-COVID-19 symptoms, will help public health professionals, biomedical scientists, and stake holders address avoidable harm caused by complex medication use.
Received: April 15, 2021

Accepted: July 3, 2021

Published: July 13, 2021

Copyright: (c) 2021 The author(s). This original article is brought to you for free and open access by ThinkIR: The University of Louisville's Institutional Repository. For more information, please contact thinkir@louisville.edu. This article is distributed under the terms of the Creative Commons Attribution 4.0 International License (CC BY 4.0), which permits unrestricted use, distribution, and reproduction in any medium, provided the original author and source are credited.

Funding Source: The author(s) received no specific funding for this work

Conflict of Interest: All authors declared no conflict of interest in relation to the main objective of this work.

\section{References}

1. Institute for Health Metrics and Evaluation. The Lancet: Latest global disease estimates reveal perfect storm of rising chronic diseases and public health failures fuelling COVID-19 pandemic. Available at: http://www.healthdata.org/ news-release/lancet-latest-global-disease-estimates-revealperfect-storm-rising-chronic-diseases-and. Accessed 17 June 2021.

2. Global burden of 369 diseases and injuries in 204 countries and territories, 1990-2019: a systematic analysis for the Global Burden of Disease Study 2019. Lancet 2020; 396(10258):1204-22. doi: 10.1016/s0140-6736(20)30925-9. PMID: 33069326.

3. Institute for Health Metrics and Evaluation. Global Burden of Disease (GBD). Available at: http://www.healthdata. org/gbd/2019. Accessed 10 April 2021.

4. Jones DS, Podolsky SH, Greene JA. The burden of disease and the changing task of medicine. $\mathrm{N}$ Engl $\mathrm{J}$ Med 2012; 366(25):2333-8. doi: 10.1056/NEJMp1113569. PMID: 22716973

5. Peach BC, Valenti M, Sole ML. A Call for the World Health Organization to Create International Classification of Disease Diagnostic Codes for Post-Intensive Care Syndrome in the Age of COVID-19. World Med Health Policy 2021. doi: 10.1002/wmh3.401. PMID: 33821196.

6. Ayoubkhani D, Khunti K, Nafilyan V, et al. Post-covid syndrome in individuals admitted to hospital with covid-19: retrospective cohort study. BMJ 2021; 372:n693. doi: 10.1136/bmj.n693. PMID: 33789877.
7. Centers for Disease Control and Prevention. Late Sequelae of COVID-19. Available at: https://www.cdc.gov/ coronavirus/2019-ncov/hcp/clinical-care/late-sequelae.html. Accessed 13 March 2021.

8. Johansson $M$, Ståhlberg $M$, Runold $M$, et al. LongHaul Post-COVID-19 Symptoms Presenting as a Variant of Postural Orthostatic Tachycardia Syndrome: The Swedish Experience. JACC Case Rep 2021; 3(4):573-80. doi: 10.1016/j.jaccas.2021.01.009. PMID: 33723532.

9. Emamikhah $M$, Babadi $M$, Mehrabani $M$, et al. Opsoclonus-myoclonus syndrome, a post-infectious neurologic complication of COVID-19: case series and review of literature. J Neurovirol 2021; 27(1):26-34. doi: 10.1007/s13365-020-00941-1. PMID: 33492608.

10. Dennis A, Wamil M, Alberts J, et al. Multiorgan impairment in low-risk individuals with post-COVID-19 syndrome: a prospective, community-based study. BMJ Open 2021; 11(3):e048391. doi: 10.1136/bmjopen-2020-048391. PMID: 33785495.

11. Crimmins EM. Age-Related Vulnerability to Coronavirus Disease 2019 (COVID-19): Biological, Contextual, and Policy-Related Factors. Public Policy Aging Rep 2020; 30(4):142-6. doi: 10.1093/ppar/praa023. PMID: 33214754.

12. Mair A, Fernandez-Llimos F, Alonso A, et al. Polypharmacy Management by 2030: a patient safety challenge. Coimbra: SIMPATHY Consortium, 2017. 
13. Centers for Disease Control and Prevention. Adverse Drug Events in Adults. Available at: https://www.cdc.gov/ medicationsafety/adult_adversedrugevents.html. Accessed 10 April 2021.

14. Nightingale G, Hajjar E, Swartz K, Andrel-Sendecki J, Chapman A. Evaluation of a pharmacist-led medication assessment used to identify prevalence of and associations with polypharmacy and potentially inappropriate medication use among ambulatory senior adults with cancer. J Clin Oncol 2015; 33(13):1453-9. doi: 10.1200/jco.2014.58.7550. PMID: 25800766 .

15. American Cancer Society. Special Section: Cancer in the Oldest Old. Available at: https://www cancer.org/content/dam/cancer-org/research/cancer-factsand-statistics/annual-cancer-facts-and-figures/2019/cancerfacts-and-figures-special-section-cancer-in-the-oldest-old2019.pdf. Accessed 10 April 2021.

16. Bektas A, Schurman SH, Franceschi C, Ferrucci L. A public health perspective of aging: do hyper-inflammatory syndromes such as COVID-19, SARS, ARDS, cytokine storm syndrome, and post-ICU syndrome accelerate short- and long-term inflammaging? Immun Ageing 2020; 17:Article 23. doi: 10.1186/s12979-020-00196-8. PMID: 32849908.

17. Antimisiaris D, McHolan B, Moga D, Mospan C. Depression Part 3: Medication Related Problems. Sr Care Pharm 2021; 36(2):68-82. doi: 10.4140/TCP.n.2021.68. PMID: 33509330

18. Antimisiaris D, Polivka B, Folz R, Myers J, Gopalraj RJ. Are protocols for medication holds sufficient for skin prick testing for older adults with asthma? Innov Aging 2017; 1(suppl_1):909-. doi: 10.1093/geroni/igx004.3257.

19. Cavallazzi RS, Polivka BJ, Beatty BL, et al. Current Bronchodilator Responsiveness Criteria Underestimate Asthma in Older Adults. Respir Care 2020; 65(8):1104-11. doi: 10.4187/respcare.07132. PMID: 32071132.
20. Carroll $E$, Neumann $H$, Aguero-Rosenfeld ME, et al. Post-COVID-19 inflammatory syndrome manifesting as refractory status epilepticus. Epilepsia 2020; 61(10):e135-e9. doi: 10.1111/epi.16683. PMID: 32944946.

21. Jamali $M$, Jaffar $H$, Ullah I, Orsolini L. COVID-19 and Cognitive, Emotional Aspects of Post-Intensive Care Syndrome. J Nerv Ment Dis 2021; 209(4):242-3. doi: 10.1097/nmd.0000000000001294. PMID: 33764952.

22. Home - US Deprescribing Research Network. Available at: https://deprescribingresearch.org/. Accessed 12 April 2021.

23. Deprescribing.org - Optimizing Medication Use. Available at: https://deprescribing.org/. Accessed 12 April 2021.

24. Australian Deprescribing Network. Available at: https: //www.australiandeprescribingnetwork.com.au. Accessed 1 May 2020.

25. Watanabe JH, Mclnnis T, Hirsch JD. Cost of Prescription Drug-Related Morbidity and Mortality. Ann Pharmacother 2018; 52(9):829-37. doi: 10.1177/1060028018765159. PMID: 29577766.

26. Centers for Disease Control and Prevention. Deaths and Mortality. Available at: https://www.cdc.gov/nchs/fastats/ deaths.htm. Accessed 10 April 2021.

27. Gurwitz JH, Field TS, Harrold LR, et al. Incidence and preventability of adverse drug events among older persons in the ambulatory setting. JAMA 2003; 289(9):1107-16. doi: 10.1001/jama.289.9.1107. PMID: 12622580.

28. Steinman MA, Handler SM, Gurwitz JH, Schiff GD, Covinsky KE. Beyond the prescription: medication monitoring and adverse drug events in older adults. $\mathrm{J} \mathrm{Am}$ Geriatr Soc 2011; 59(8):1513-20. doi: 10.1111/j.15325415.2011.03500.x. PMID: 21797831. 